\title{
Initial Experience of Consecutive Robotic Pancreatoduodenectomies With Patient Reported Outcomes From a Single Center in the UK
}

\author{
Omar Jalila, Qazi Rahim Muhammad ${ }^{\mathrm{a}}$, Alexia Farrugia ${ }^{\mathrm{b}, \mathrm{c}}$, Majid Alia ${ }^{\mathrm{a}}$ Paschalis Gavriilidis ${ }^{\mathrm{a}, \mathrm{d}}$, \\ Gabriele Marangoni ${ }^{\mathrm{a}, \mathrm{c}}$, Jawad Ahmada, c, d
}

\section{To the Editor}

Robotic surgery has seen exponential growth in the UK during the last 5 years. In fact, in the country, only two centers (including ours) perform robotic pancreatoduodenectomies (RPDs). The aim of the present study was to evaluate and report the postoperative outcomes and patient satisfaction of the first 16 consecutive RPDs from a single center. Prospectively collected data for RPDs were analyzed for postoperative outcomes. All the operations were performed with the Da Vinci surgical system (Intuitive Surgical Inc., Sunnyvale, CA, USA).

All operations were performed using the same technique. In particular, pancreatojejunostomies were performed according to modified Blumgart technique using 3-0 proline on $31 \mathrm{~mm}$ needle for the outer layer and 5-0 PDS on $17 \mathrm{~mm}$ needle for duct to mucosa anastomosis [1]. Hepaticojejunostomy was performed using 5-0 PDS continuous anterior and posterior layers when the duct was more than $1 \mathrm{~cm}$ and interrupted sutures in a smaller duct.

The International Study Group on Pancreatic Surgery (ISGPS) definitions of the post-pancreatectomy fistula, hemorrhage, delayed gastric emptying, and Dindo-Clavien classification were used to categorize the postoperative complications $[2,3]$. A validated fistula risk score calculator was used to assess the risk for development of postoperative pancreatic fistula [4]. Patient satisfaction was assessed using the RAND 36-Item Health survey [5]. To prevent performance bias, a junior Doctor from a different team who had no prior knowledge

Manuscript submitted August 1, 2021, accepted August 7, 2021

Published online August 30, 2021

a University Hospitals Coventry and Warwickshire NHS Trust, Clifford Bridge Road, Coventry CV2 2DX, UK

${ }^{b}$ Walsall Healthcare NHS Trust, Walsall Manor Hospital, Moat Road, Walsall WS2 9PS, UK

${ }^{c}$ Warwick Medical School, University of Warwick, Medical School Building, Coventry CV4 7AL, UK

${ }^{\mathrm{d} C o r r e s p o n d i n g ~ A u t h o r: ~ J a w a d ~ A h m a d, ~ U n i v e r s i t y ~ H o s p i t a l s ~ C o v e n t r y ~ a n d ~}$ Warwickshire NHS Trust, Clifford Bridge Road, Coventry CV2 2DX, UK. Email: Jawad.ahmad@uhcw.nhs.uk; Paschalis Gavriilidis, University Hospitals Coventry and Warwickshire NHS Trust, Clifford Bridge Road, Coventry CV2 2DX, UK. Email: pgavrielidis@yahoo.com

doi: https://doi.org/10.14740/jocmr4574 of patient's pre- and postoperative details conducted the interviews. The patients rated at discharge, 2 weeks and 6 months postoperatively.

All continuous data were presented as median or mean \pm standard deviation (SD) and all categorical variables were presented as numbers and percentages.

Twelve of 16 patients were included in the analysis. Two operations were converted to open, one due to extend malignant portal vein involvement with a view to vein resection and the other due to failure to progress due to high body mass index (BMI). Another two operations were abandoned, one due to peritoneal metastases and the other with intraoperative possible diagnosis of liver cirrhosis.

Mean operative time was $547 \pm 65 \mathrm{~min}$ and the mean intraoperative blood loss was $245 \pm 119 \mathrm{~mL}$. Docking and undocking time was included in the operative time and was about 30 $\min$.

Eleven out of 12 patients belong to American Society of Anesthesiologists (ASA) tier II and the mean BMI was 24.9 \pm 2.8 . Obviously, the above parameters had a positive impact on the postoperative recovery. Only two out of 12 patients developed Dindo-Clavien IIIb complications. Although, four $(33 \%)$ out of 12 patients were characterized as of high risk for occurrence of postoperative fistula; none of the patients developed grade $\mathrm{B}$ or $\mathrm{C}$ fistula, only one patient was diagnosed with grade A fistula (Table 1).

Only two (17\%) out of 12 patients were diagnosed with R1 margins.

The low incidence rates of major complications and pancreatic fistula obviously reflected on the shorter hospital stay compared with open procedures performed by the same team. The mean hospital stay was $8.33 \pm 2.8$ days (Table 1 ).

Although the number of enthusiastic supporters of RPD has increased recently exponentially, there are limited data reported on patient satisfaction and the associated quality of life (QoL) following robotic surgery $[1,6-8]$. The present study is one of the first reporting QoL. Notably, only one patient out of 12 complained of severe non-specific body pains at discharge and 2 weeks postoperatively (Table 2).

Both parameters of physical functioning score and general health score demonstrated an increase compared to baseline score; these findings provide evidence for better QoL. In addition, the patients score higher for social functioning. In par- 
Table 1. Patient Characteristics, Intra- and Postoperative Outcomes, Postoperative Pain Scores

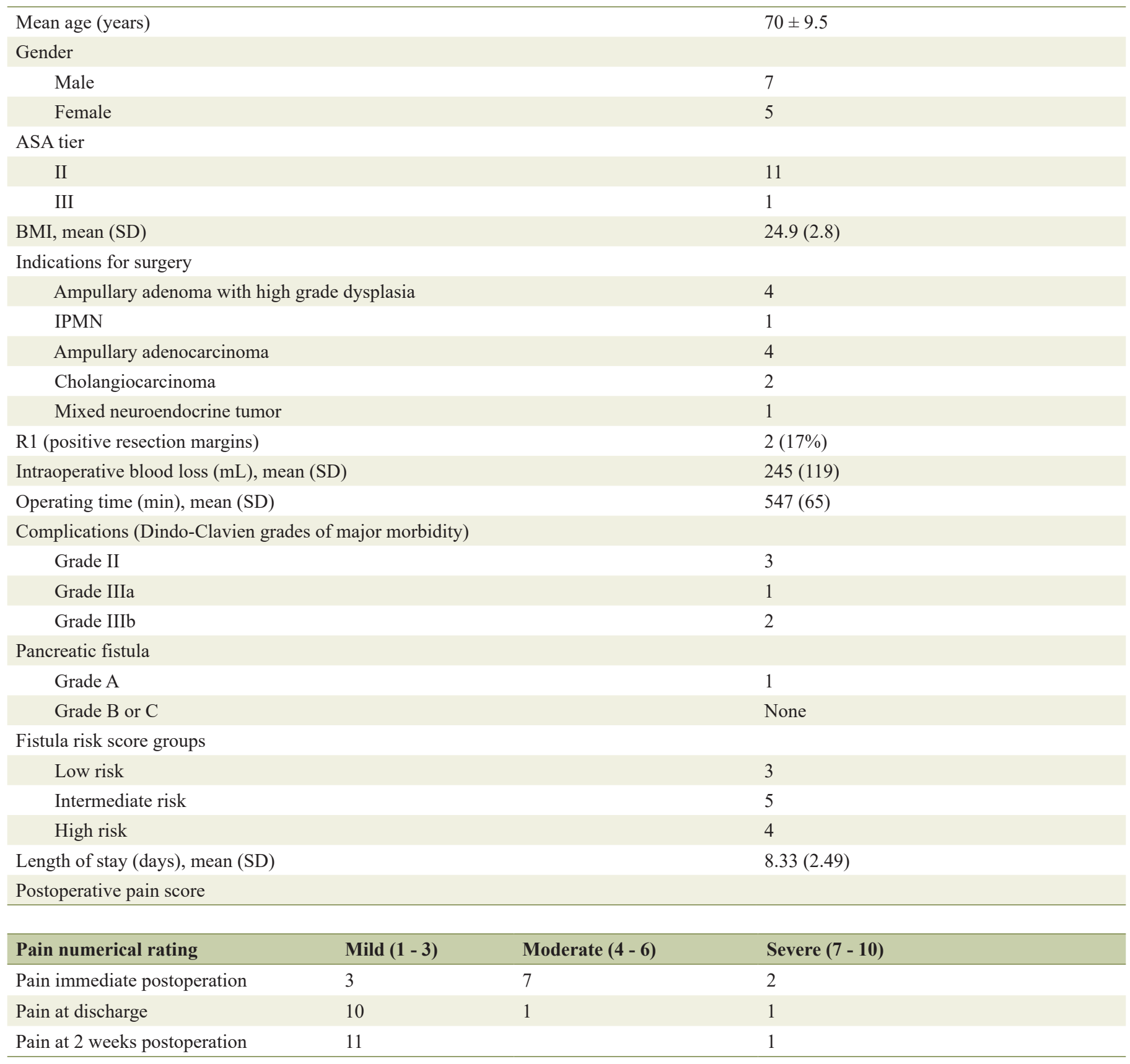

ASA: American Society of Anesthesiologists; BMI: body mass index; SD: standard deviation; IPMN: intraductal papillary mucinous neoplasm.

ticular, 10 patients reported that it took them up to 5 weeks to recover since operation and remaining two recovered within 5 - 10 weeks (Table 2).

To the author's best knowledge, this is the first study in the UK reporting postoperative outcomes and QoL in patients undergoing RPD. It demonstrates the feasibility, safety and efficacy of the RPD; moreover, it demonstrates high patient satisfaction for major operation such as pancreatoduodenectomy. Results from comparative studies and data from international registries will certainly help health care providers further to support robotic pancreatic surgery in the future. However, the results of the present study should be interpreted in the context of its limitations. The data are from initial experience from a single center in the UK. Therefore, institutional and underpowered sample bias might have influenced the results.

\section{Acknowledgments}

None to declare. 
Table 2. Quality of Life Survey and Postoperative Pain Score

\begin{tabular}{lll}
\hline & Pre-operative scores, mean (SD) & Postoperative scores, mean (SD) \\
\hline $\begin{array}{l}\text { SF-36 survey domains } \\
\text { Physical functioning }\end{array}$ & $83(12)$ & $90(9)$ \\
General health & $38(7.2)$ & $70(7.5)$ \\
Bodily pain & $84(9.5)$ \\
\hline $\begin{array}{l}\text { Social functioning } \\
\text { Other condition-specific symptoms }\end{array}$ & 96 \\
$\begin{array}{l}\text { Feeling motivated } \\
\text { Feeling depressed }\end{array}$ & Yes: $11 ;$ No: 1 \\
\hline $\begin{array}{l}\text { Full of energy } \\
\text { Charge in emotional well-being }\end{array}$ & Yes: 1 ; No: 11 \\
\hline
\end{tabular}

SD: standard deviation.

\section{Financial Disclosure}

None to declare.

\section{Conflict of Interest}

None to declare.

\section{Informed Consent}

Not applicable.

\section{Author Contributions}

OJ: investigation, methodology, validation, writing original draft, review; QRM: investigation, methodology, validation, writing original draft, review; AF: investigation, methodology, validation, writing original draft, review; MA: investigation, methodology, validation, writing original draft, review; PG: investigation, analysis, methodology, validation, writing original draft, review; GM: investigation, methodology, validation, writing original draft, review; JA: conceptualization, analysis, investigation, methodology, validation, writing original draft, review, supervision.

\section{Data Availability}

The authors declare that data supporting the findings of this study are available within the article.

\section{References}

1. Wang SE, Chen SC, Shyr BU, Shyr YM. Comparison of Modified Blumgart pancreaticojejunostomy and pancreaticogastrostomy after pancreaticoduodenectomy. HPB (Oxford). 2016;18(3):229-235.

2. Bassi C, Marchegiani G, Dervenis C, Sarr M, Abu Hilal M, Adham M, Allen P, et al. The 2016 update of the International Study Group (ISGPS) definition and grading of postoperative pancreatic fistula: 11 Years After. Surgery. 2017;161(3):584-591.

3. Dindo D, Demartines N, Clavien PA. Classification of surgical complications: a new proposal with evaluation in a cohort of 6336 patients and results of a survey. Ann Surg. 2004;240(2):205-213.

4. Callery MP, Pratt WB, Kent TS, Chaikof EL, Vollmer $\mathrm{CM}$, Jr. A prospectively validated clinical risk score accurately predicts pancreatic fistula after pancreatoduodenectomy. J Am Coll Surg. 2013;216(1):1-14.

5. Hays RD, Sherbourne CD, Mazel RM. The RAND 36item health survey 1.0. Health Econ. 1993;2(3):217-227.

6. Arms RG, 3rd, Sun CC, Burzawa JK, Fleming ND, Nick AM, Rallapalli V, Westin SN, et al. Improvement in quality of life after robotic surgery results in patient satisfaction. Gynecol Oncol. 2015;138(3):727-730.

7. Shyr BU, Shyr BS, Chen SC, Chang IW, Shyr YM, Wang SE. Operative results and patient satisfaction after robotic pancreaticoduodenectomy. Asian J Surg. 2020;43(4):519525.

8. Patel BY, White L, Gavriilidis P, Satyadas T, Frampton AE, Pai M. A systematic review into patient reported outcomes following pancreaticoduodenectomy for malignancy. Eur J Surg Oncol. 2021;47(5):970-978. 